\title{
On visual pigment templates and the spectral shape of invertebrate rhodopsins and metarhodopsins
}

\author{
Doekele G. Stavenga
}

Received: 2 July 2010/Revised: 3 August 2010/Accepted: 5 August 2010/Published online: 20 August 2010

(C) The Author(s) 2010. This article is published with open access at Springerlink.com

\begin{abstract}
The absorbance spectra of visual pigments can be approximated with mathematical expressions using as single parameter the absorbance peak wavelength. A comparison of the formulae of Stavenga et al. in Vision Res 33:1011-1017 (1993) and Govardovskii et al. in Vis Neurosci 17:509-528 (2000) applied to a number of invertebrate rhodopsins reveals that both templates well describe the normalized $\alpha$-band of rhodopsins with peak wavelength $>400 \mathrm{~nm}$; the template spectra are virtually indistinguishable in an absorbance range of about three log units. The template formulae of Govardovskii et al. in Vis Neurosci 17:509-528 (2000) describe the rhodopsin spectra better for absorbances below $10^{-3}$. The template predicted spectra deviate in the ultraviolet wavelength range from each other as well as from measured spectra, preventing a definite conclusion about the spectral shape in the wavelength range $<400 \mathrm{~nm}$. The metarhodopsin spectra of blowfly and fruitfly R1-6 photoreceptors derived from measured data appear to be virtually identical. The established templates describe the spectral shape of fly metarhodopsin reasonably well. However, the best fitting template spectrum slightly deviates from the experimental spectra near the peak and in the long-wavelength tail. Improved formulae for fitting the fly metarhodopsin spectra are proposed.
\end{abstract}

Keywords Blowfly - Drosophila - Ultraviolet . Absorbance difference spectra $\cdot$ Photoequilibrium

D. G. Stavenga $(\varangle)$

Department of Neurobiophysics, University of Groningen, Groningen, The Netherlands

e-mail: D.G.Stavenga@rug.nl

\section{Introduction}

Vision starts with the absorption of photons by the visual pigment (rhodopsin) molecules. This causes conversion of the rhodopsin molecules into the so-called active metarhodopsin state, which then, by coupling to a G-protein, triggers the phototransduction process. The metarhodopsin is inactivated by binding to an arrestin molecule, which stops the phototransduction machinery. In the photoreceptors of vertebrates, the metarhodopsins rapidly, thermally decay into a separate opsin protein and chromophore, and hence the possible photoreconversion of metarhodopsin into the native rhodopsin is in normal light conditions negligible; the visual pigment content of vertebrate photoreceptors therefore is maintained by a highly involved enzymatic renewal process (Saari 2000). In contrast with this, the metarhodopsins of invertebrate photoreceptors are thermostable (Hamdorf 1979). The metarhodopsin-both the active and inactive state-can also absorb a photon, which then results in regeneration of the rhodopsin, eventually after release of the bound arrestin and dephosphorylation of rhodopsin. Photoreconversion of metarhodopsin is therefore considered to be a prominent pathway for visual pigment maintenance in invertebrates. Nevertheless, invertebrates renew their visual pigment molecules also (Schwemer 1984; Goldsmith and Bernard 1985), but like in vertebrates, this process is slow, taking several minutes to hours (Schwemer 1989).

Intense and prolonged illumination of invertebrate photoreceptors results in a photo-equilibrium of their visual pigments, where the rhodopsin to metarhodopsin concentration ratio is determined by the absorbance spectra and quantum efficiencies of the two visual pigment states and the spectral composition of the illuminant. Consequently, in studies of invertebrate phototransduction, not only the 
spectral properties of the rhodopsin but also those of the metarhodopsin have to be known, specifically when concerning the interaction of arrestin with metarhodopsin (Belušič et al. 2010; Satoh et al. 2010). The aim of the present paper was to update the current knowledge of invertebrate visual pigment spectra.

The absorbance spectra of vertebrate rhodopsins known half a century ago inspired Dartnall (1953) to devise a nomogram, from which the spectral shape of any rhodopsin could be derived using only one parameter, the peak wavelength, $\lambda_{\text {max }}$. This lead was followed by several other investigators, notably Mansfield and MacNichol (MacNichol 1986), who demonstrated that the spectra of vertebrate visual pigments have the same shape when plotted on an inverse wavelength scale. The two classes of visual pigments based on vitamin $\mathrm{A}_{1}$ and vitamin $\mathrm{A}_{2}$ each appeared to have a specific invariant shape.

For both the vitamin $\mathrm{A}_{1}$ - and $\mathrm{A}_{2}$-based visual pigments, Stavenga et al. (1993) developed modified lognormal formulae, with free parameter $\lambda_{\text {max }}$, that well described the shape of experimental absorbance spectra plotted on a linear ordinate scale. Absorbance measurements can usually be accurately measured over about two log units of magnitude, but photoreceptor spectral sensitivities can often be conveniently measured over several log units. Lamb (1995) therefore devised a new template that covered a large sensitivity range. Govardovskii et al. (2000) elaborated this approach further by fitting the spectra of several vertebrate visual pigments, thus achieving a now widely used set of formulae.

Concerning the visual pigments of invertebrates, Lipetz and Cronin (1988) found that experimentally measured spectra of crustacean rhodopsins were well described by the vitamin $A_{1}$ template of vertebrate visual pigments, but not by the template of vitamin $\mathrm{A}_{2}$ visual pigments, and thus they suggested that retinal is the chromophore of crustacean rhodopsins. Vertebrate and invertebrate visual pigments apparently follow very similar spectral rules, and the templates initially developed for vertebrate rhodopsins thus are now used by both researchers of vertebrate and invertebrate vision.

Assuming that the rhodopsin template also holds for metarhodopsins, metarhodopsin spectra have been derived from absorbance difference measurements on isolated invertebrate eyes (e.g., moth: Langer et al. 1979; crayfish: Cronin and Goldsmith 1982; fruitfly: Salcedo et al. 1999; Kiselev et al. 2000; firefly: Cronin et al. 2000). Here, we reconsider the rhodopsin and metarhodopsin absorbance spectra of a number of invertebrate species and specifically investigate whether the R1-6 photoreceptors of the blowfly Calliphora and Drosophila have metarhodopsins with the same invariant spectral shape as that of the rhodopsins. It appears that the fly metarhodopsin absorbance spectra slightly deviate from the spectrum predicted by the rhodopsin template.

\section{Materials and methods}

Rhodopsin absorbance spectrum

The absorbance spectra of rhodopsins consist of bands, called the $\alpha$-band, $\beta$-band, etc. (Fig. 1). In experimental investigations often only the $\alpha$-band is measured. Its spectral shape is well described by (Stavenga et al. 1993)

$S_{\alpha}=\exp \left\{-a x^{2}\left(\left[1+b x+3 / 8(b x)^{2}\right]\right\}\right.$,

with $x=\log _{10}\left(\lambda / \lambda_{\max }\right) ; \lambda$ is the wavelength and $\lambda_{\max }$ the peak wavelength of the $\alpha$-band. For vitamin $\mathrm{A}_{1}$-based pigments $a=380$ and $b=6.09$. The $\beta$-band, assumed to have a fixed peak wavelength at $350 \mathrm{~nm}$, was described with the same expression (Eq. 1), but with relative amplitude $A_{\beta}=0.29$, and $a=247$ and $b=3.59$ (Stavenga et al. 1993). Instead of the two parameters of Eq. 1, the expression for the $\alpha$-band of Govardovskii et al. (2000) employs seven parameters:

$S_{\alpha}=\{\exp [A(a-x)]+\exp [B(b-x)]+\exp [C(c-x)]+D\}^{-1}$,

with $x=\left(\lambda / \lambda_{\max }\right)^{-1}$ and $A=69.7, a=0.8795+0.0459$ $\exp \left[-\left(\lambda_{\max }-300\right)^{2} / 11940\right], B=28, b=0.922, C=-14.9$, $c=1.104, D=0.674$. For the $\beta$-band, Govardovskii et al. (2000) conceived a Gaussian function, $S_{\beta}=A_{\beta} \exp$ $\left\{-\left[\left(\lambda-\lambda_{\beta}\right) / d\right]^{2}\right\}$, with relative amplitude $A_{\beta}=0.26$, peak wavelength $\lambda_{\beta}=189+0.315 \lambda_{\max }$ and width parameter $d=-40.5+0.195 \lambda_{\max }$.

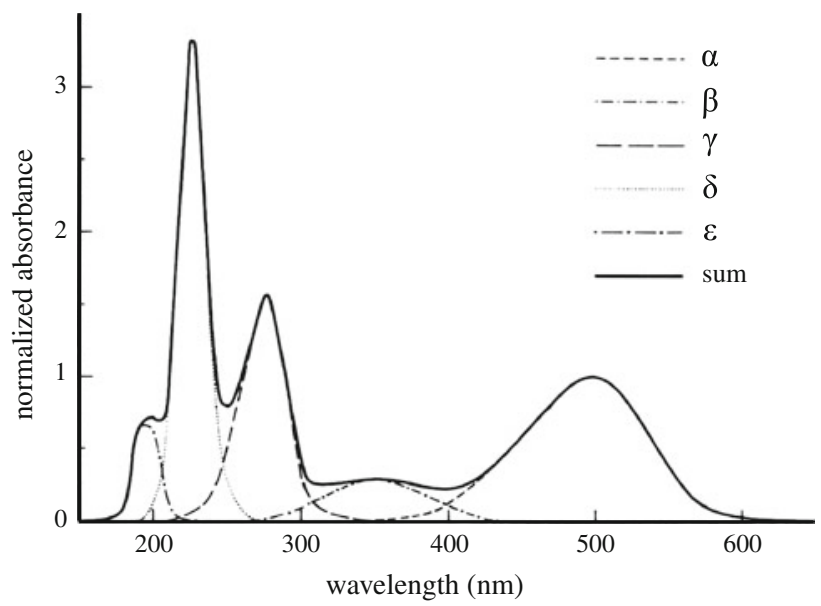

Fig. 1 The absorbance spectrum of an exemplary vertebrate rhodopsin, considered as a sum of absorbance bands, indicated by $\alpha, \beta$, etcetera, normalized to the peak absorbance of the $\alpha$-band (after Stavenga and Van Barneveld 1975) 
As an example, Fig. 2 presents the theoretical spectra of visual pigments with $\lambda_{\max }=350,450$, and $550 \mathrm{~nm}$ following from the template formulae of Stavenga et al. (1993; SSH) and Govardovskii et al. (2000; Gov). The spectra plotted on a linear ordinate scale appear to be virtually indistinguishable in the visual wavelength range, but they deviate in the ultraviolet (Fig. 2a). The spectra also differ for very small absorbances, as is seen in the plots on a logarithmic ordinate scale (Fig. 2b).

Photochemistry of invertebrate visual pigments

The photochemistry of invertebrate visual pigments is described by the scheme $\mathrm{R} \Leftrightarrow \mathrm{M}$. The rate constants $k_{\mathrm{R}}$ and $k_{\mathrm{M}}$ for the photoconversions of $\mathrm{R}$ to $\mathrm{M}$ and of $\mathrm{M}$ to $\mathrm{R}$, respectively, are $k_{i}=\int \beta_{i}(\lambda) I(\lambda) d \lambda$, with $i=\mathrm{R}, \mathrm{M} ; \beta_{\mathrm{R}}(\lambda)=$ $\gamma_{\mathrm{R}} \alpha_{\mathrm{R}}(\lambda)$ and $\beta_{\mathrm{M}}(\lambda)=\gamma_{\mathrm{M}} \alpha_{\mathrm{M}}(\lambda)$ are the photosensitivities, with $\alpha_{R}(\lambda)$ and $\alpha_{M}(\lambda)$ the molecular absorbance coefficients of rhodopsin and metarhodopsin, and $\gamma_{R}$ and $\gamma_{M}$ the quantum efficiencies for photoconversion; $I(\lambda)$ is the photon flux. Continuous illumination of the visual pigment establishes a photoequilibrium or photosteady state with a metarhodopsin fraction $f_{\mathrm{M}, \mathrm{e}}=1 /\left(1+k_{\mathrm{M}} / k_{\mathrm{R}}\right)$. In the case of monochromatic illumination $f_{\mathrm{M}, \mathrm{e}}(\lambda)=1 /\left[1+\varphi \alpha_{\mathrm{M}}(\lambda) / \alpha_{\mathrm{R}}(\lambda)\right]$, with
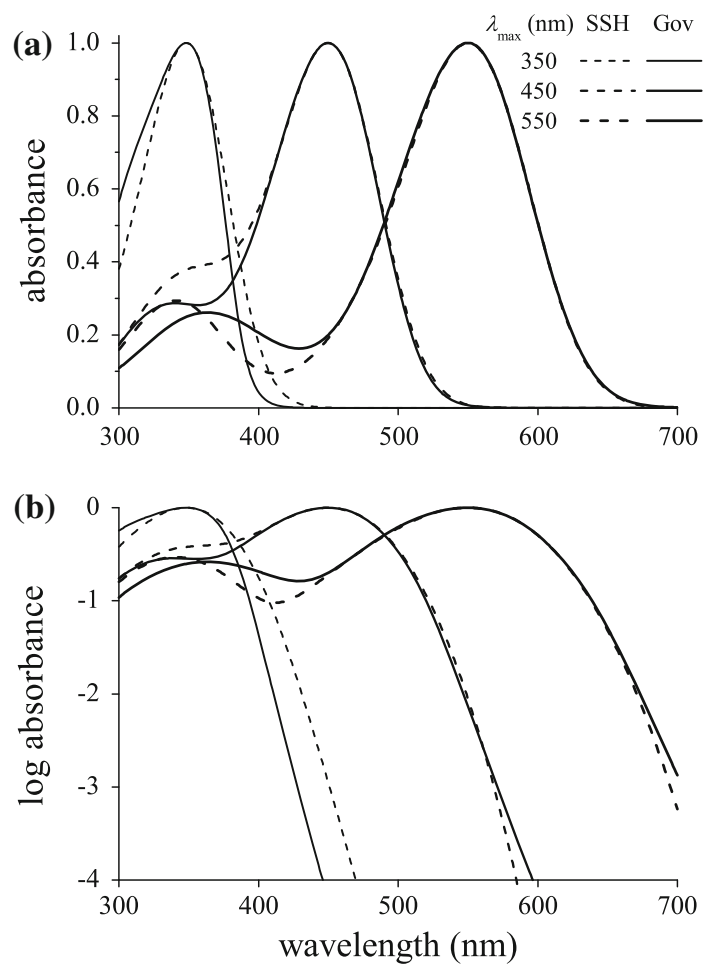

Fig. 2 Theoretical spectra of visual pigments with $\alpha$-bands peaking at 350,450 , and $550 \mathrm{~nm}$ following from the template formulae of Stavenga et al. (1993; SSH) and Govardovskii et al. (2000; Gov), plotted on a linear (a) and logarithmic (b) ordinate scale. The spectra deviate in the short-wavelength range and when the absorbance becomes very small
$\varphi=\gamma_{\mathrm{M}} / \gamma_{\mathrm{R}}$ the relative quantum efficiency (see e.g., Stavenga and Schwemer 1984; Stavenga et al. 2000). If the visual pigment is illuminated with a constant photon flux $I(\lambda)$, but initially not in photoequilibrium, then the metarhodopsin fraction (and of course also the rhodopsin fraction) changes with a time constant $\tau=\left(k_{\mathrm{R}}+k_{\mathrm{M}}\right)^{-1}$ (Hochstein et al. 1978). For monochromatic light $\tau(\lambda)=\left[\left\{\beta_{\mathrm{R}}(\lambda)+\right.\right.$ $\left.\left.\beta_{\mathrm{M}}(\lambda)\right\} I(\lambda)\right]^{-1}$, and therefore the relaxation spectrum $\beta_{\text {rel }}(\lambda)=\beta_{\mathrm{R}}(\lambda)+\beta_{\mathrm{M}}(\lambda)$ can be determined by measuring the applied photon flux and the resulting time constant of photoconversion as a function of wavelength.

\section{Absorbance difference spectra}

Measurement of visual pigment spectra in the native eye tissue is often cumbersome due to contamination by additional pigments and other disturbing optical effects. These effects can be eliminated, at least partly, by calculating the absorbance difference spectrum between two different photochemically induced cases. The difference spectra measured on the eyes of the blowfly Calliphora vicina (formerly C. erythrocephala) by Schwemer (1979) and the fruitfly Drosophila melanogaster by Kiselev et al. (2000) were used, together with template-predicted rhodopsin spectra, for calculating the metarhodopsin spectrum of the R1-6 photoreceptors of the two fly species.

\section{Results}

Rhodopsin and metarhodopsin spectra of various invertebrates

The rhodopsin absorbance spectrum is the main determinant for the spectral sensitivity of visual photoreceptors. Höglund et al. (1973) characterized three photoreceptor types in the superposition eye of the sphingid moth Deilephila elpenor by measuring the electroretinogram (ERG) and applying selective adaptation. They compared the spectral sensitivities with spectra following from the Dartnall nomogram, yielding estimates for the photoreceptors' peak wavelengths: 350, 440, and $525 \mathrm{~nm}$ (Fig. 3 of Höglund et al. 1973; see also Fig. 20 of Hamdorf 1979). Only the spectrum of the green receptor corresponded well with the expected spectrum, but the spectral sensitivities of the blue and ultraviolet receptor strongly deviated from the spectra derived from the nomogram. However, the template formulae devised since the publication date of the moth spectra by Stavenga et al. (1993; SSH) and Govardovskii et al. (2000; Gov) approximate the measurements rather well (Fig. 3a). Optimal fits were obtained with peak wavelengths 357,441 , and $524 \mathrm{~nm}$. Neither of the templates shows a clear fit in the ultraviolet, however, meaning 

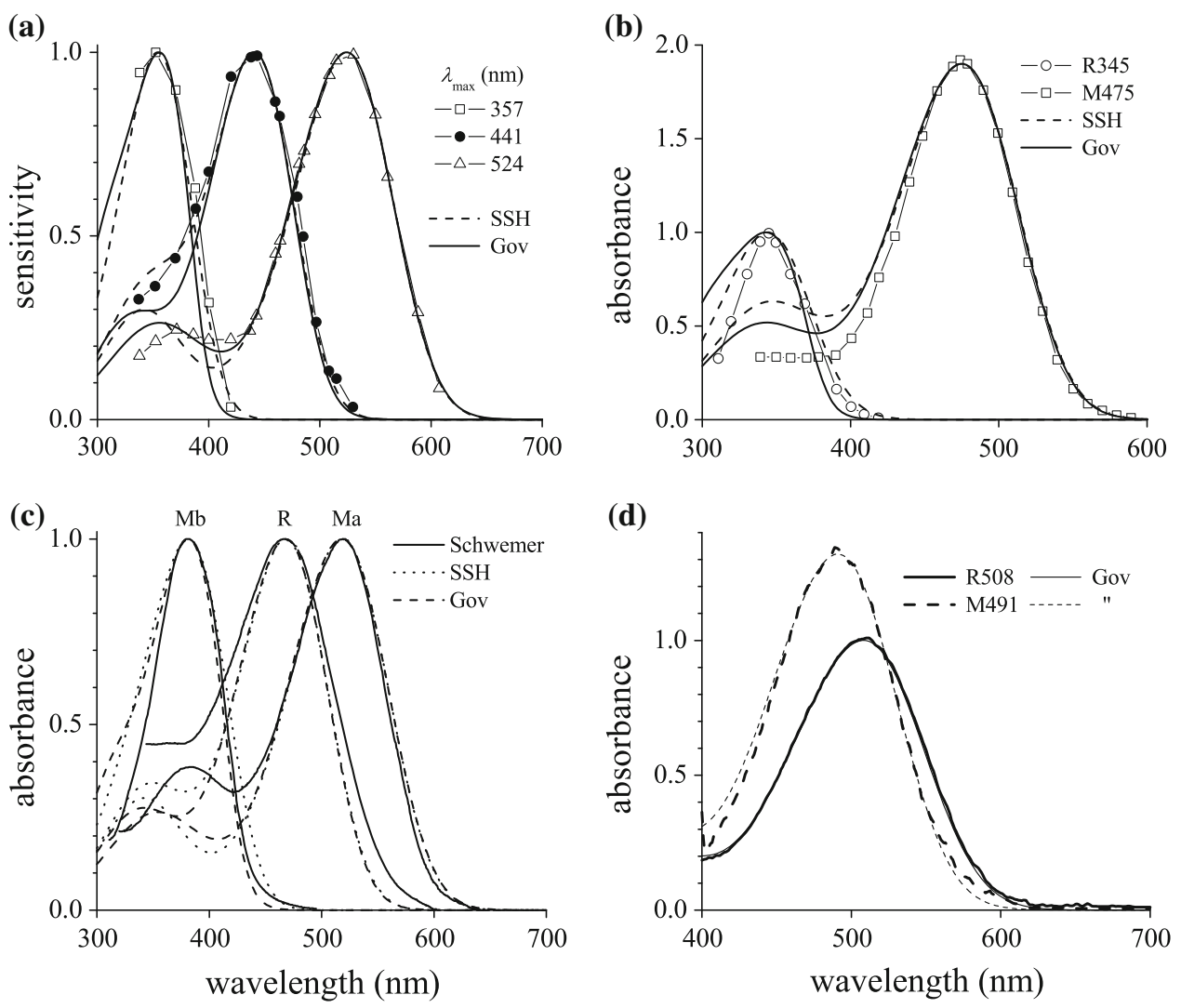

Fig. 3 Spectra representing invertebrate rhodopsins and metarhodopsins. a Sensitivity spectra of the three photoreceptor types of the sphingid moth Deilephila elpenor measured by electroretinography and selective adaptation (symbols; from Fig. 3 of Höglund et al. 1973), together with visual pigment absorbance spectra with peak wavelength 357, 441 and $524 \mathrm{~nm}$. b Absorbance spectra for the UVrhodopsin (R345) and its metarhodopsin (M475), normalized at the rhodopsin peak, derived from visual pigment extracts of eyes of the owlfly Ascalaphus macaronius (symbols; from Fig. 4 of Hamdorf

that either the experimental data are contaminated or the templates are inadequate in the short wavelength range.

For studying the photochemistry of ultraviolet absorbing rhodopsins without possible contamination of other visual pigments, the owlfly Ascalaphus macaronius, with its UV-receptor dominated retina, has shown to be of outstanding historical significance. Figure $3 \mathrm{~b}$ shows the spectra of the UV-rhodopsin and its metarhodopsin, which have peak wavelengths at 345 and $475 \mathrm{~nm}$, respectively (Hamdorf et al. 1973; symbols from their Fig. 4; see also Hamdorf 1979, Fig. 6), together with template-predicted curves. The metarhodopsin (M475) has a distinctly ( 1.9 times) higher peak absorbance than the rhodopsin (R345). The longwavelength limb of the metarhodopsin spectrum is well fitted by both templates, but in the ultraviolet wavelength range the correspondence is unsatisfactory. The SSH-template approximates the UV rhodopsin (R345) slightly better than the Gov-template, but both templates fail to accurately reproduce the short wavelength flank. Investigation of the

et al. 1973). c Normalized absorbance spectra of the rhodopsin (R) and its acid metarhodopsin (Ma) and basic metarhodopsin (Mb) of the octopus Eledone moschata (from Schwemer 1969). d Spectra of the rhodopsin (R508) and metarhodopsin (M491) of the hermit crab Petrochirus diogenes, normalized at the rhodopsin peak (from Fig. 2c of Cronin and Forward 1988). Only fits with the Gov-template formulae are shown, because the curves following from the SSH- and Gov-template were virtually identical

UV-sensitive $7 \mathrm{p}$ photoreceptor of the housefly, Musca domestica, yielding an R330 (Fig. 3 of Hardie and Kirschfeld 1983), gave a result similar to that of the Ascalaphus UV rhodopsin: the SSH-template fits better than the Gov-template (not shown).

Figure $3 \mathrm{c}$ shows the case of the blue-absorbing rhodopsin of the cephalopod Eledone moschata (Schwemer 1969). Photon absorption converts the rhodopsin (R468) at physiological $\mathrm{pH}$ into the so-called acid metarhodopsin state (Ma518), but strong alkalinization transforms it into the basic metarhodopsin (Mb382) state. The experimental spectra are shown here normalized, but as with all other known invertebrate visual pigments, the metarhodopsins absorb stronger than the rhodopsin (Hamdorf 1979). It appears that the rhodopsin spectrum is not well described by the templates. Yet, the long-wavelength limbs of both metarhodopsins are closely approximated by the template curves, but the correspondences in the ultraviolet range are again not very satisfactory. 


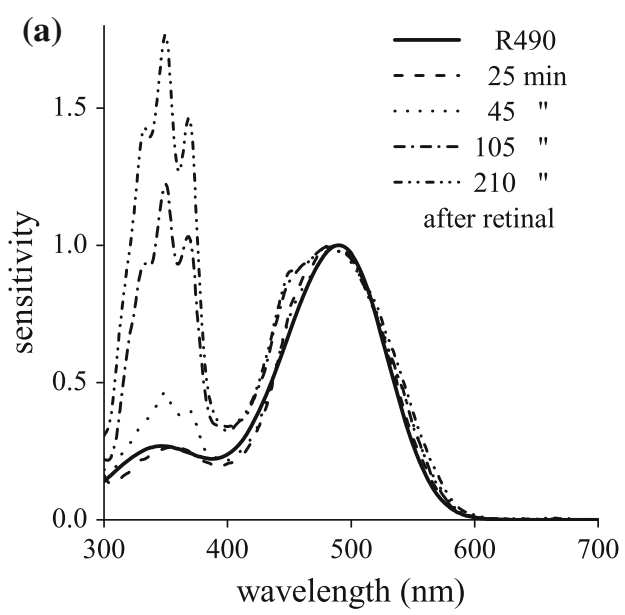

Fig. 4 Sensitivity spectra of blowfly R1-6 photoreceptors. a Spectral sensitivities measured by intracellular recording after administering a drop of retinal to the eye of flies that were reared on a vitamin A poor diet (from Hamdorf et al. 1992, Fig. 5). After $25 \pm 5$ min the spectral sensitivity is very similar to the absorbance spectrum of a visual pigment peaking at $490 \mathrm{~nm}$ ( $\mathrm{R} 490$; calculated with the Govtemplate). The progressive increase of the sensitivity in the ultraviolet after $45 \pm 5 \mathrm{~min}, 105 \pm 15 \mathrm{~min}$, and $210 \pm 60 \mathrm{~min}$ indicates the incorporation of sensitizing pigment. b Photoreceptor spectral sensitivities calculated with a model for the integrated optical system of

Figure $3 \mathrm{~d}$ presents the spectra of the green-absorbing rhodopsin of the hermit crab Petrochirus diogenes and its blue-absorbing metarhodopsin (Cronin and Forward 1988). The experimental data, given for wavelengths $>400 \mathrm{~nm}$, are well approximated by both the SSH- and Gov-template with peak wavelengths 508 and $491 \mathrm{~nm}$. The curves following from the SSH- and Gov-template were virtually identical, and therefore only the Gov-curves are presented in Fig. 3d; also in the cases treated below where both templates give indistinguishable curves (out of courtesy to Govardovskii) only the Gov-results are presented.

The examples of Fig. 3 show that in most cases the $\alpha$-band of the rhodopsins equally well conforms with the predictions of the SSH- and Gov-template. However, in the ultraviolet the situation is less favorable. The expression for the $\beta$-band of the SSH-template confounds the expression for the $\alpha$-band, resulting for $350<\lambda_{\max }<400 \mathrm{~nm}$ in spectra with an absorbance peak wavelength a few nm less than the given $\lambda_{\max }$-value. For the Gov-template a similar bathochromic shift occurs for $\lambda_{\max }<350 \mathrm{~nm}$. Nevertheless, the template predictions strongly differ in the ultraviolet, even after correction for the peak wavelength, and appear to be inaccurate in the UV. In other words, in the ultraviolet the templates are inadequate. Unfortunately, the data available in the literature do not seem to be sufficiently accurate to construct an improved template. The next section shows that the ultraviolet wavelength range is especially complicated for fly photoreceptors.

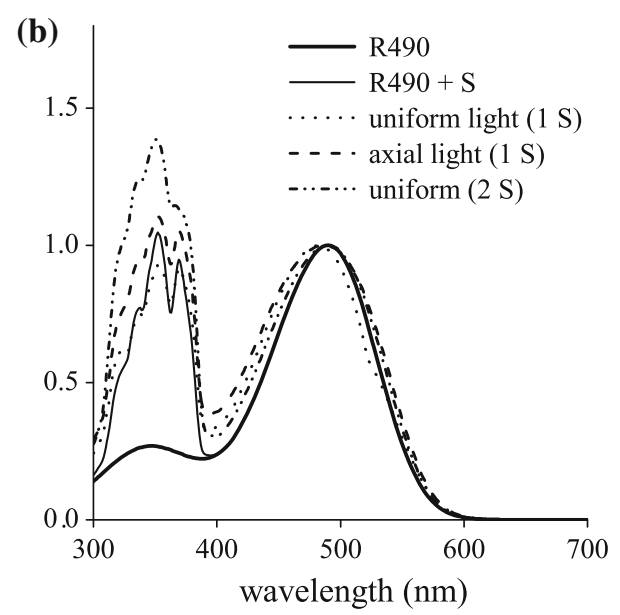

the fly facet lens and rhabdomere, using a facet lens diameter $31 \mu \mathrm{m}$, a rhabdomere with diameter $1.6 \mu \mathrm{m}$ and length $250 \mu \mathrm{m}$, and a peak absorbance coefficient $0.006 \mu \mathrm{m}^{-1}$ of the R490 rhodopsin, the absorption of which was enhanced in the UV by the sensitizing pigment $\mathrm{S}$. The normalized sensitivity spectra slightly depend on whether the fly eye is illuminated with an axial point source or a uniform light source, and when one $(1 \mathrm{~S})$ or two $(2 \mathrm{~S})$ sensitizing molecules are assumed to sensitize the rhodopsin molecule (from Stavenga 2004a, Fig. 9, and Stavenga 2004b, Fig. 5)

Spectral sensitivity of blowfly photoreceptors

Approximation of an experimentally determined sensitivity spectrum with a rhodopsin template spectrum must be executed with caution, because several factors can modulate the spectral sensitivity, as is demonstrated in Fig. 4a. Hamdorf et al. (1992) recorded from photoreceptors of blowflies Calliphora vicina reared on a vitamin A-deprived diet. A blue-green-peaking spectral sensitivity was obtained (Fig. 4a, curve $25 \mathrm{~min}$ ) that can be well approximated with the absorbance spectrum of an $\mathrm{A}_{1}$-rhodopsin with absorbance maximum at $490 \mathrm{~nm}$ (R490; Fig. 4a). Application of all-trans retinal to the eye produced an increase in light sensitivity and, most strikingly, the spectra showed a gradual increase of the ultraviolet sensitivity relative to the blue-green sensitivity. The three-peaked fine structure (Fig. 4a, curves 45, 105, $210 \mathrm{~min}$ ) was identified to be due to the binding of 3-hydroxy-retinol to the rhodopsin, resulting in sensitization for UV absorption by the rhodopsin, which has 3-hydroxy-retinal as the chromophore (Kirschfeld et al. 1977; Vogt and Kirschfeld 1983). The spectra also showed an asymmetric broadening of the main, $\alpha$-band (Fig. 4a, curves 105 and $210 \mathrm{~min}$ ).

Optical calculations of the spectral sensitivity of a blowfly photoreceptor in situ containing an R490 rhodopsin demonstrated that the broadening of the spectra is a direct consequence of the integrated optical system of facet lens and rhabdomere waveguide and, furthermore, that the 
precise shape of the sensitivity spectrum depends on the illumination, being for example an axial point source or a uniform, wide field light source (Fig. 4b; from Stavenga $2004 a$, b). In other words, experimentally obtained sensitivity spectra can severely deviate from the underlying rhodopsin spectrum.

\section{Absorbance spectra of blowfly rhodopsin} and metarhodopsin

Schwemer (1979) extracted visual pigment from blowfly retinae and measured transmission spectra before and after bleaching. He thus obtained an absorbance difference spectrum with a long-wavelength band that closely fitted an R490 (Gov) template spectrum (Fig. 5a). Transmission measurements of a blowfly retina illuminated with red and blue light, respectively, yielded a spectrum (Fig. 5b, dif) representing the (normalized) absorbance difference between an orange-absorbing metarhodopsin and the R490 rhodopsin (Schwemer 1979). Interestingly, the UV part of the difference spectrum featured undulations, reminiscent of the three peaks in the electrophysiological measurements of the photoreceptor spectral sensitivity (Fig. 4a). The negative absorbance difference in the ultraviolet indicates that the UV-sensitization of the metarhodopsin is less than that of the rhodopsin. Also, the absorbance of the metarhodopsin without the sensitizing pigment may be lower in the UV than the rhodopsin absorbance.

If the normalized absorbance coefficient of rhodopsin is $\alpha_{\mathrm{R}}^{*}(\lambda)$, and the normalized difference spectrum is $\delta(\lambda)$, then the absorbance coefficient of the metarhodopsin spectrum follows from $\alpha_{\mathrm{M}}^{*}(\lambda)=\alpha_{\mathrm{R}}^{*}(\lambda)+v \delta(\lambda)$, where $v$ is an unknown parameter. The ratio between the metarhodopsin and rhodopsin peak absorbance becomes 1.6, 1.7, and 1.8,
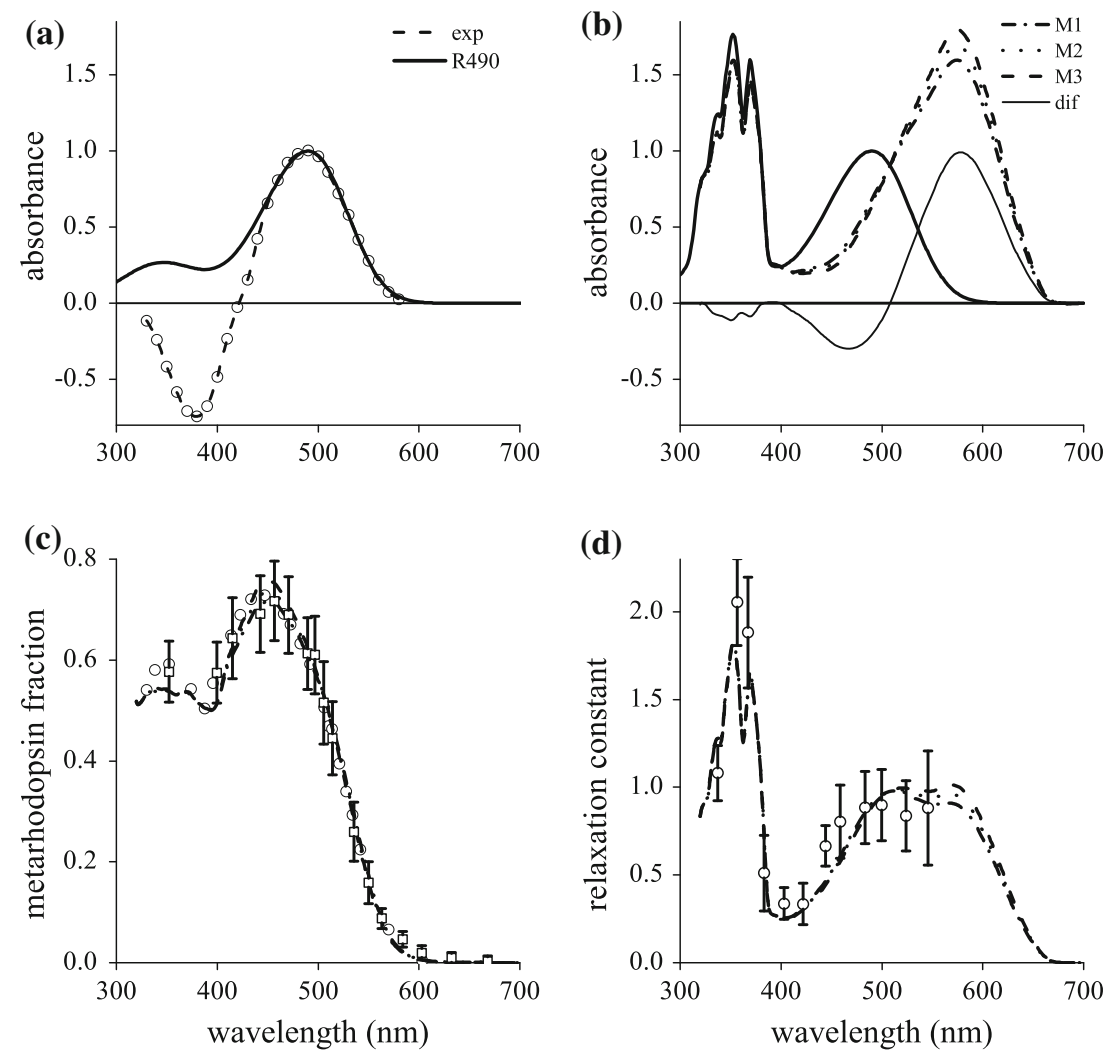

Fig. 5 Spectral characteristics of the visual pigment in R1-6 photoreceptors of the blowfly Calliphora vicina. a Absorbance difference spectrum of an extract of blowfly retinae and its bleaching product (exp; from Schwemer 1979) fitted with the Gov-template for a rhodopsin with peak wavelength $490 \mathrm{~nm}$ (R490). b Absorbance spectrum of the combined rhodopsin R490 and sensitizing pigment, normalized at the $490 \mathrm{~nm}$ peak (bold continuous curve; after Fig. 4), the absorbance difference spectrum of the metarhodopsin and rhodopsin state measured from isolated retinae (dif; from Schwemer 1979), and three metarhodopsin spectra, obtained by adding to the sensitized R490 spectrum the normalized difference spectrum

multiplied with a factor 1.56, 1.66, and 1.76. The resulting metarhodopsin spectra have a peak wavelength at $575 \mathrm{~nm}$ and (relative) peak absorbance coefficients of 1.6 (M1), 1.7 (M2), and 1.8 (M3). c Metarhodopsin fraction in photoequilibrium created by monochromatic light (circles from Schwemer 1979; squares plus error bars from Stavenga et al. 1973) and spectra calculated with the rhodopsin and metarhodopsin spectra of (b), using a relative quantum efficiency value 0.95 (dotted/dashed curves). d Relaxation spectra (symbols from Minke and Kirschfeld 1979) compared with spectra calculated using the spectra of (b); see Methods 
respectively, when taking $v=1.56,1.66$, and 1.76, yielding the three dotted/dashed spectra of Fig. 5b. The resulting metarhodopsin spectra have all a peak wavelength at about $575 \mathrm{~nm}$, virtually independent of the parameter $v$, because of the severely bathochromic-shifted metarhodopsin peak. Using the relative quantum efficiency value $\varphi=0.95$, estimated for blowfly R1-6 rhodopsin (Schwemer 1979), photoequilibrium spectra, $f_{\mathrm{M}, \mathrm{e}}(\lambda)$, can be calculated from the rhodopsin and metarhodopsin spectra of Fig. 5b (dotted/dashed curves in Fig. 5c; see Methods). The calculated spectra correspond well with experimental photoequilibrium spectra (Fig. 5c, symbols; from Stavenga et al. 1973 and Schwemer 1979). A similar correspondence holds for the calculated normalized relaxation spectra (see Methods) and the spectrum measured by Minke and Kirschfeld (1979; Fig. 5d). However, the accuracy of the experimental spectra is insufficient to decide between the different calculated metarhodopsin spectra of Fig. 5b.

An alternative, direct method for estimating the metarhodopsin spectrum is via fluorescence (crayfish; Cronin and Goldsmith 1981). Fly metarhodopsin features a distinct fluorescence (Stavenga 1983), but in addition a special, highly fluorescing visual pigment state, called M', can be populated by intense UV/blue illumination (Franceschini et al. 1981). Its excitation and emission spectra were first measured in houseflies (Musca domestica; Stavenga et al. 1984). Subsequently, very similar spectra were obtained for both $\mathrm{M}$ and $\mathrm{M}^{\prime}$ in the blowfly Calliphora vicina (Kruizinga and Stavenga 1990). Figure 6 presents the excitation and emission spectra of blowfly $\mathrm{M}^{\prime}$ together with the three calculated metarhodopsin spectra of Fig. 5b normalized at the long-wavelength peak.

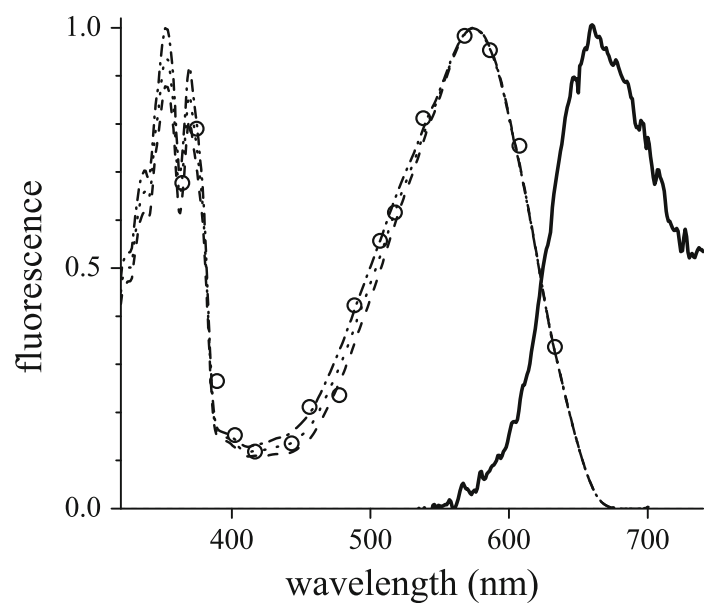

Fig. 6 Metarhodopsin fluorescence spectra. The symbols and continuous curve are the experimentally determined excitation values and emission spectrum of the metarhodopsin state $\mathrm{M}^{\prime}$ (from Kruizinga and Stavenga 1990). The dashed, dotted, and dash-dotted curves are the normalized metarhodopsin spectra of Fig. 5b
Absorbance spectra of fruitfly rhodopsin and metarhodopsin

The absorbance spectrum of the R1-6 rhodopsin of the fruitfly, Drosophila, Rh1, has a peak wavelength at about $480 \mathrm{~nm}$, as follows from both optical measurements on isolated eyes and extracts and photoreceptor spectral sensitivities (Stark et al. 1977; Ostroy 1978; Salcedo et al. 1999). Figure 7a presents the absorbance spectrum of R480 together with the normalized difference spectrum measured on visual pigment extracts from fruitfly heads (Kiselev et al. 2000). By combining the rhodopsin spectrum and the difference spectrum in the same way as done for blowfly metarhodopsin (Fig. 5b), and using the same three values for the parameter $v(1.56,1.66$, and 1.76), three absorbance spectra for fruitfly metarhodopsin, all with peak wavelength at about $575 \mathrm{~nm}$, are obtained.

The sets of metarhodopsin spectra calculated for Calliphora (Fig. 5b) and Drosophila (Fig. 7a) appear to be virtually identical. Figure $7 \mathrm{~b}$ presents the Calliphora and Drosophila spectra having a relative peak absorbance 1.8 with respect to their rhodopsin peak together with a metarhodopsin spectrum predicted by a best fitting Govardovskii-rhodopsin template with peak wavelength $570 \mathrm{~nm}$ and peak height value 1.8. The template-predicted spectrum approximates the calculated metarhodopsin spectra reasonably well, but distinct differences occur near the peak and the long-wavelength tail. The template spectrum has a hypsochromic-shifted peak wavelength (from 575 to $570 \mathrm{~nm}$ ) and a much shallower long-wavelength tail.

\section{Discussion}

In vision research it has become common practice to apply visual pigment template formulae so as to heuristically estimate the shape and peak wavelength of photoreceptor spectral sensitivities. The well-fitting templates for moth (Fig. 3a) and blowfly (Fig. 4a, 5a) sensitivity spectra might suggest that these insects have vitamin $\mathrm{A}_{1}$ visual pigments. However, biochemical analyses have shown that the chromophore of lepidopteran and fly visual pigments is 3-hydroxyretinal (Vogt 1989). 3-hydroxyretinol is called vitamin $\mathrm{A}_{3}$, and because it is a xanthophyll derivative, Vogt has proposed xanthopsin as the common term for a vitamin $A_{3}$-based visual pigment (Vogt 1989). The name xanthopsin is, however, nowadays often used for the photoactive yellow protein of eubacterial blue-light photoreceptors (Perman et al. 1998). Until further nomenclature decisions are made, it seems preferable to use rhodopsin as the generic name for the visual pigments.

The template formulae for vitamin $\mathrm{A}_{1}$-based rhodopsins of Stavenga et al. (1993; SSH) as well as that of 

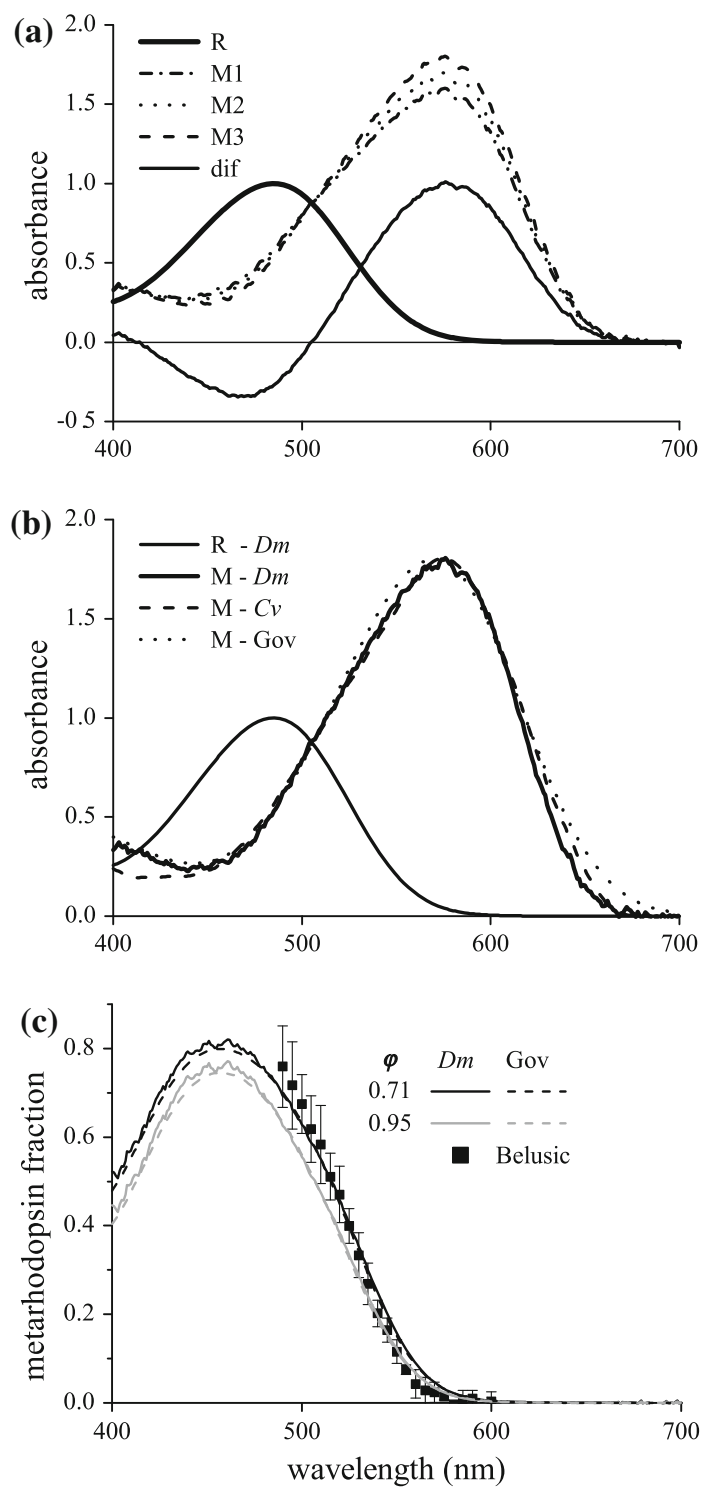

Fig. 7 Rhodopsin and metarhodopsin spectra of the fruitfly Drosophila melanogaster. a Drosophila rhodopsin (R; R480 calculated with the Gov-template), the normalized absorbance difference spectrum (dif) measured by Kiselev et al. (2000), and three metarhodopsin spectra obtained by adding to the rhodopsin spectrum the difference spectrum multiplied by a factor 1.56, 1.66, and 1.76. The resulting metarhodopsin spectra have peak wavelengths at $575 \mathrm{~nm}$ and (relative) peak absorbance coefficients of 1.6 (M1), 1.7 (M2), and 1.8 (M3). b Drosophila rhodopsin (R-Dm) and metarhodopsin with relative peak value $1.8(\mathrm{M}-\mathrm{Dm})$, compared with the Calliphora metarhodopsin spectrum (M- $C v ; \mathrm{M} 3$ of Fig. 5b), together with a closely fitting spectrum following from the Gov-template (M-Gov). c Metarhodopsin fraction in photoequilibrium as a function of the adapting wavelength, calculated for relative quantum efficiencies $\varphi=0.71$ and 0.95 , using R-Dm and M-Dm of (b) and R-Dm and M-Gov of (b), respectively, and experimental data obtained by fluorescence measurements (from Belušič et al. 2010)

Govardovskii et al. (2000; Gov) are presently used in many photoreceptor studies, both on vertebrates and invertebrates. The expressions for the vitamin $\mathrm{A}_{1}$-based rhodopsins are also used for the vitamin $\mathrm{A}_{3}$-based rhodopsins of insects, because the two different chromophores when combined with the same opsin probably yield very similar absorbance spectra. Considering the achievable accuracy of experimental data, the predicted spectra for the $\alpha$-band are indeed indistinguishable for rhodopsins with peak wavelength $\lambda_{\max }>400 \mathrm{~nm}$ when plotted on a linear scale. Because of its simpler form, the SSH-template may be easier to use, for instance, in fitting procedures. For fitting spectral sensitivities covering more than three log units, the Gov-template must be used.

The formula for the $\beta$-band with a fixed peak wavelength given by Stavenga et al. (1993) is certainly of limited value because later work has demonstrated that the peak wavelength of the $\beta$-band is correlated with that of the $\alpha$-band (Palacios et al. 1996). However, whether the expression for the $\beta$-band given by Govardovskii et al. (2000) is adequate for all $A_{1}$ - and $A_{3}$-based rhodopsins may be questionable (see e.g., the present Fig. 3, and also Fig. 7 of Govardovskii et al. 2000). For rhodopsins with $\lambda_{\max }<400 \mathrm{~nm}$, the templates noticeably deviate from each other. Unfortunately, the present experimental data are not fully consistent with either of the templates (see the present Fig. 3b, and also Fig. 5 of Govardovskii et al. 2000). More carefully measured data are clearly necessary to clarify these deviations.

Salcedo et al. (1999) performed electroretinography (ERG) on the fruitfly Drosophila and obtained for the R1-6 photoreceptors a spectral sensitivity peaking at $\sim 475 \mathrm{~nm}$, together with a fine-structured spectral sensitivity in the ultraviolet with an amplitude twice as high as that of the $\alpha$-band. Similar data reported by previous workers (see, e.g., Stark et al. 1977) underscore the similarity of the spectral properties of fruitfly and blowfly R1-6 photoreceptors, except for the slightly blue-shifted spectral sensitivity of fruitfly photoreceptors. Salcedo et al. (1999) furthermore derived absorbance difference spectra from microspectrophotometrical measurements on isolated fruitfly eyes. Using the SSH visual pigment template, they fitted the R1-6 difference spectrum with a rhodopsin and metarhodopsin pair, R486 and M566. Using these data, Belušič et al. (2010), in their study on the role of arrestin in Drosophila phototransduction, could satisfactorily describe the photoequilibrium spectrum determined in Drosophila eyes via metarhodopsin fluorescence measurements (Fig. 7c).

Yet, the spectra derived in Fig. $7 \mathrm{~b}$ indicate that the data of Salcedo et al. (1999) may have to be slightly corrected. Taking the average metarhodopsin spectrum derived for Calliphora and Drosophila and fitting that with an SSHtemplate for rhodopsin yielded a peak wavelength of about $570 \mathrm{~nm}$; the spectra derived from measured difference spectra (Fig. 7b) peak at $575 \mathrm{~nm}$, however. A better fit to the fly metarhodopsin spectra can be obtained by using for the 


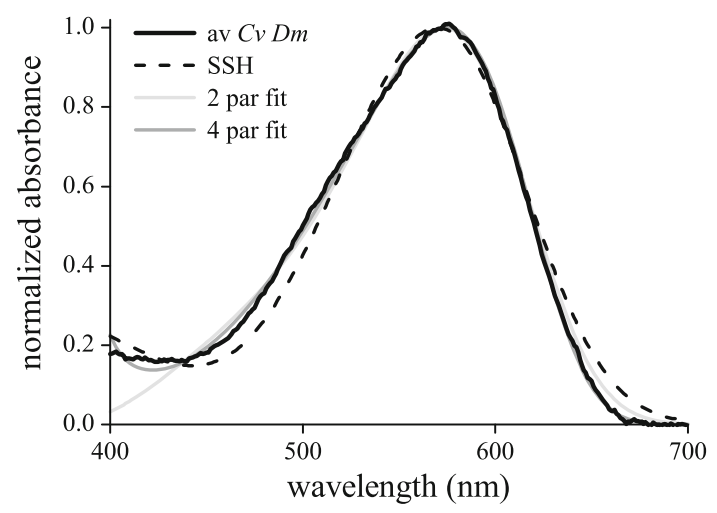

Fig. 8 Average of the metarhodopsin spectra derived in Fig. 7b for blowfly (Calliphora vicina, $C v$ ) and fruitfly (Drosophila melanogaster, $\mathrm{Dm}$ ) compared with spectra following from the SSH-template and from modified SSH-templates with two and four parameters

two parameters $a$ and $b$ in the SSH-template expression $S_{\alpha}=\exp \left\{-a x^{2}\left(\left[1+b x+3 / 8(b x)^{2}\right]\right\}\right.$ the values $a=396$ and $b=10.1$, yielding $\lambda_{\max }=573 \mathrm{~nm}$. A further improved fit is achieved with the slightly modified expression $S_{\alpha}=\exp \left\{-a x^{2}\left(\left[1+b x+c x^{2}+d x^{3}\right]\right\}\right.$ using four parameters, $a=387, b=14.0, c=120$, and $d=418$, with $\lambda_{\max }=575 \mathrm{~nm}$. The various spectra are combined in Fig. 8 .

The retinal chromophores of the rhodopsins and metarhodopsins are in the 11-cis and all-trans configuration, respectively, and therefore the $\alpha$-band of the metarhodopsins is higher than that of the rhodopsin. The $\alpha$-bands of the spectra of the (acid) metarhodopsins of octopus (Fig. 3c) and owlfly (Fig. 3d) are well approximated by the current template formulae, but the fly metarhodopsin spectra clearly exhibit slight deviations. The observed differences in spectral shape, which must be due to the differences in interactions of the chromophore with the opsin moiety, may stimulate further photophysical studies of invertebrate metarhodopsins.

The differences in the chromophore configurations will also have consequences for the $\beta$-band, and therefore the rhodopsin template formulae will most likely yield aberrant spectra in the ultraviolet wavelength range. More detailed spectra, for a broader range of visual pigments, will be necessary to decide to what extent template formulae can be used to describe metarhodopsin spectra. Special attention is needed for the ultraviolet range, where reliable measurements for metarhodopsins are generally lacking. In the case of flies, the sensitizing pigment complicates the spectrum in the UV, and for most other cases the ultraviolet range has been poorly sampled, or the ultraviolet was contaminated by various photoproducts.

Accurate knowledge of the spectral shapes of the rhodopsin-metarhodopsin pair may become important in phototransduction studies focused on understanding the control function of arrestin, which binds active metarhodopsin (Belušič et al. 2010; Satoh et al. 2010). Figure $7 \mathrm{c}$ presents measurements of the dependence of the metarhodopsin fraction in photoequilibrium on the wavelength of the adapting light together with values calculated for Drosophila rhodopsin R-Dm and the metarhodopsin spectra of Fig. 7b (M-Dm and M-Gov). Two values of the relative quantum efficiency $\varphi$ were used: the. $\varphi=0.71$ was found for Drosophila visual pigment by Stark and Johnson (1980), in fair agreement with values obtained for other invertebrate visual pigments (see Table 4 of Stavenga and Schwemer 1984), but $\varphi=0.95$ was estimated for the blowfly Calliphora by Schwemer (1979). At any rate, for both relative quantum efficiency values, the metarhodopsin fraction is virtually identical for the two metarhodopsin spectra. The metarhodopsin fraction spectra are clearly also not strongly dependent on the relative quantum efficiency value.

\section{Conclusion}

The templates for visual pigment spectra are now established tools in both vertebrate and invertebrate vision research. Given the accuracy of the vast majority of experimental cases where absorbance and/or sensitivity spectra are measured and where the peak wavelength of the $\alpha$-band is $>400 \mathrm{~nm}$, the SSH- and Gov-templates are equally applicable. There is an urgent need for better spectral data in the ultraviolet, both for enhancing our knowledge of the spectral shape of the UV-rhodopsins as well as that of the $\beta$-band of the rhodopsins with $\lambda_{\max }>400 \mathrm{~nm}$. The shape of the absorbance spectrum of the metarhodopsins probably slightly deviates from that of the rhodopsins, but when applied with some caution, for a first approach the standard templates may be useful for finding the peak wavelength of the $\alpha$-band, at least for calculating the metarhodopsin fraction in photoequilibrium.

Acknowledgments I thank Drs Kentaro Arikawa, Tom Cronin, Roger Hardie, and Primoz Pirih for reading the manuscript and providing valuable suggestions. Financial support was given by AFOSR/ EOARD grant no. FA8655-08-1-3012.

Open Access This article is distributed under the terms of the Creative Commons Attribution Noncommercial License which permits any noncommercial use, distribution, and reproduction in any medium, provided the original author(s) and source are credited.

\section{References}

Belušič G, Pirih P, Stavenga DG (2010) Photoreceptor responses of fruitflies with normal and reduced arrestin content studied by simultaneous measurements of visual pigment fluorescence and ERG. J Comp Physiol A 196:23-35 
Cronin TW, Forward RB (1988) The visual pigments of crabs. I. Spectral characteristics. J Comp Physiol A 162:463-478

Cronin TW, Goldsmith TH (1981) Fluorescence of crayfish metarhodopsin studied in single rhabdoms. Biophys J 35:653-664

Cronin TW, Goldsmith TH (1982) Quantum efficiency and photosensitivity of the rhodopsin-metarhodopsin conversion in crayfish photoreceptors. Photochem Photobiol 36:447-454

Cronin TW, Järvilehto M, Weckström M, Lall AB (2000) Tuning of photoreceptor spectral sensitivity in fireflies (Coleoptera: Lampyridae). J Comp Physiol A 186:1-12

Dartnall HJA (1953) The interpretation of spectral sensitivity curves. Brit Med Bull 9:24-30

Franceschini N, Kirschfeld K, Minke B (1981) Fluorescence of photoreceptor cells observed in vivo. Science 213:1264-1267

Goldsmith TH, Bernard GD (1985) Visual pigments of invertebrates. Photochem Photobiol 42:805-809

Govardovskii VI, Fyhrquist N, Reuter T, Kuzmin DG, Donner K (2000) In search of the visual pigment template. Vis Neurosci 17:509-528

Hamdorf K (1979) The physiology of invertebrate visual pigments. In: Autrum H (ed) Handb Sens Physiol Vol VII/6A, pp 145-224. Springer, Berlin

Hamdorf K, Paulsen R, Schwemer J (1973) Photoregeneration and sensitivity control of photoreceptors of invertebrates. In: Langer $\mathrm{H}$ (ed) Biochemistry and physiology of visual pigments. Springer, Berlin, pp 155-166

Hamdorf K, Hochstrate P, Höglund G, Moser M, Sperber S, Schlecht $P$ (1992) Ultra-violet sensitizing pigment in blowfly photoreceptors R1-6; probable nature and binding sites. J Comp Physiol A 171:601-615

Hardie RC, Kirschfeld K (1983) Ultraviolet sensitivity of fly photoreceptors R7 and R8: evidence for a sensitising function. Biophys Struct Mech 9:171-180

Hochstein S, Minke B, Hillman P, Knight BW (1978) The kinetics of visual pigments. I. Mathematical analysis. Biol Cybern 30:23-32

Höglund G, Hamdorf K, Langer H, Paulsen R, Schwemer J (1973) The photopigments in an insect retina. In: Langer $\mathrm{H}$ (ed) Biochemistry and physiology of visual pigments. Springer, Berlin, pp 167-174

Kirschfeld K, Franceschini N, Minke B (1977) Evidence for a sensitising pigment in fly photoreceptors. Nature 269:386-390

Kiselev A, Socolich M, Vinos J, Hardy RW, Zuker CS, Ranganathan R (2000) A molecular pathway for light-dependent photoreceptor apoptosis in Drosophila. Neuron 28:139-152

Kruizinga B, Stavenga DG (1990) Fluorescence spectra of blowfly metaxanthopsins. Photochem Photobiol 51:197-201

Lamb TD (1995) Photoreceptor spectral sensitivities: common shape in the long-wavelength region. Vision Res 35:3083-3091

Langer H, Hamann B, Meinecke CC (1979) Tetrachromatic visual system in the moth Spodoptera exempta (Insecta, Noctuidae). J Comp Physiol 129:235-239

Lipetz LE, Cronin TW (1988) Application of an invariant spectral form to the visual pigments of crustaceans-Implications regarding the binding of the chromophore. Vision Res 28:1083-1093

MacNichol EF Jr (1986) A unifying presentation of photopigment spectra. Vision Res 26:1543-1556

Minke B, Kirschfeld K (1979) The contribution of a sensitizing pigment to the photosensitivity spectra of fly rhodopsin and metarhodopsin. J Gen Physiol 73:517-540

Ostroy SE (1978) Characteristics of Drosophila rhodopsin in wild-type and norpA vision transduction mutants. J Gen Physiol 72:717-732

Palacios AG, Goldsmith TH, Bernard GD (1996) Sensitivity of cones from a cyprinid fish (Danio aequipinnatus) to ultraviolet and visible light. Vis Neurosci 13:411-421
Perman B, Srajer V, Ren Z, Teng T, Pradervand C, Ursby T, Bourgeois D, Schotte F, Wulff M, Kort R, Hellingwerf K, Moffat K (1998) Energy transduction on the nanosecond time scale: early structural events in a xanthopsin photocycle. Science 279:1946-1950

Saari JC (2000) Biochemistry of visual pigment regeneration. Invest Ophthalmol Vis Sci 41:337-348

Salcedo E, Huber A, Henrich S, Chadwell LV, Chou WH, Paulsen R, Britt SG (1999) Blue- and green-absorbing visual pigments of Drosophila: ectopic expression and physiological characterization of the R8 photoreceptor cell-specific Rh5 and Rh6 rhodopsins. J Neurosci 19:10716-10726

Satoh AK, Xia H, Yan L, Hardie RC, Ready DF (2010) Arrestin translocation is stoichiometric to rhodopsin isomerization and accelerated by phototransduction in Drosophila photoreceptors. Neuron (in press)

Schwemer J (1969) Der Sehfarbstoff von Eledone moschata und seine Umsetzungen in der lebenden Netzhaut. Z Vergl Physiol 62:121-152

Schwemer J (1979) Molekulare Grundlagen der Photorezeption bei der Schmeissfliege Calliphora erythrocephala Meig. Habilitationsschrift, Bochum

Schwemer J (1984) Renewal of visual pigment in photoreceptors of the blowfly. J Comp Physiol A 154:535-547

Schwemer J (1989) Visual pigments of compound eyes-structure, photochemistry, and regeneration. In: Stavenga DG, Hardie RC (eds) Facets of vision. Springer, Berlin Heidelberg, pp 112-133

Stark WS, Johnson MA (1980) Microspectrophotometry of Drosophila visual pigment: determinations of conversion efficiency in R1-6 photoreceptors. J Comp Physiol 140:275-286

Stark WS, Ivanyshyn AM, Greenberg RM (1977) Sensitivity of photopigments of R1-6, a two-peaked photoreceptor in Drosophila, Calliphora and Musca. J Comp Physiol A 121:289-305

Stavenga DG (1983) Fluorescence of blowfly metarhodopsin. Biophys Struct Mech 9:309-317

Stavenga DG (2004a) Angular and spectral sensitivity of fly photoreceptors. III. Dependence on the pupil mechanism in the blowfly Calliphora. J Comp Physiol A 190:115-129

Stavenga DG (2004b) Visual acuity of fly photoreceptors in natural conditions-dependence on UV sensitizing pigment and lightcontrolling pupil. J Exp Biol 207:1703-1713

Stavenga DG, Schwemer J (1984) Visual pigments of invertebrates. In: Ali MA (ed) Photoreception and vision of invertebrates. Plenum, New York, pp 11-61

Stavenga DG, Van Barneveld HH (1975) On dispersion in visual photoreceptors. Vision Res 15:1091-1095

Stavenga DG, Zantema A, Kuiper JW (1973) Rhodopsin processes and the function of the pupil mechanism in flies. In: Langer $\mathrm{H}$ (ed) Biochemistry and physiology of visual pigments. Springer, Berlin Heidelberg New York, pp 175-180

Stavenga DG, Franceschini N, Kirschfeld K (1984) Fluorescence of housefly visual pigment. Photochem Photobiol 40:653-659

Stavenga DG, Smits RP, Hoenders BJ (1993) Simple exponential functions describing the absorbance bands of visual pigment spectra. Vision Res 33:1011-1017

Stavenga DG, Oberwinkler J, Postma M (2000) Modeling primary visual processes in insect photoreceptors. In: Stavenga DG, DeGrip WJ, Pugh EN Jr (eds) Molecular mechanisms in visual transduction. Handb Biol Phys, Vol. 3. Elsevier, Amsterdam, pp 527-574

Vogt K (1989) Distribution of insect visual chromophores: functional and phylogenetic aspects. In: Stavenga DG, Hardie RC (eds) Facets of vision. Springer, Berlin, pp 134-151

Vogt K, Kirschfeld K (1983) Sensitizing pigment in the fly. Biophys Struct Mech 9:319-328 